\title{
Exciton-Polariton Mediated Superconductivity
}

\author{
Fabrice P. Laussy, ${ }^{1}$ Alexey V. Kavokin, ${ }^{1,2}$ and Ivan A. Shelykh ${ }^{3,4}$ \\ ${ }^{1}$ School of Physics and Astronomy, University of Southampton, Southampton, SO171BJ, United Kingtom \\ ${ }^{2}$ Dipartimento di Fisica, Universita' di Roma “Tor Vergata," 00133 Roma, Italy \\ ${ }^{3}$ Science Institute, University of Iceland, Dunhagi 3, IS-107, Reykjavik, Iceland \\ ${ }^{4}$ St. Petersburg Academic University, Khlopina Street, 8/3, 194021 St. Petersburg, Russia
}

(Received 15 July 2009; published 9 March 2010)

\begin{abstract}
We revisit the exciton mechanism of superconductivity in the framework of microcavity physics, replacing virtual excitons as a binding agent of Cooper pairs by excitations of an exciton-polariton BoseEinstein condensate. We consider a model microcavity where a quantum well with a two-dimensional electron gas is sandwiched between two undoped quantum wells, where a polariton condensate is formed. We show that the critical temperature for superconductivity dramatically increases with the condensate population, opening a new route towards high-temperature superconductivity.
\end{abstract}

DOI: 10.1103/PhysRevLett.104.106402

Microcavity polaritons are quasiparticles that arise from the strong coupling of microcavity photons with quantumwell (QW) excitons [1]. They have attracted considerable interest for their manifestations of quantum phenomena, from stimulated scattering [2] and polariton lasing [3] to Bose-Einstein condensation (BEC) [4] and superfluidity $[5,6]$. Their in-plane dispersion is strongly nonparabolic. Near the ground state corresponding to zero in-plane wave vector, an extremely small polariton mass-averaging the exciton mass and the much smaller cavity-photon massbrings the critical temperature for quantum degeneracy up to room temperature $[7,8]$. On the other hand, at wave vectors exceeding the wave vector of light at the exciton resonance frequency, the polariton dispersion becomes excitonlike and its effective mass exceeds that near the band minimum by 4 orders of magnitude (see Fig. 1). Exciton polaritons are electrically neutral and cannot carry electric current. However, they may coexist and interact with free electrons or holes, if these carriers are introduced in the same QW with the excitons or in a neighboring QW [9]. When confined together, exciton polaritons and free carriers form a Bose-Fermi mixture which is expected to exhibit peculiar optical and electronic properties. In this Letter, we study the possibility of superconductivity in semiconductor microcavities containing both undoped QWs and thin $n$-doped semiconductor layers. We show that exciton-polariton mediated superconductivity is possible.

Conventional superconductivity occurs at low temperatures and can be described within the framework of BCS theory [10], which relies on the formation of Cooper pairs. Following the discovery of a phonon-mediated attraction between electrons [11], Cooper found that two electrons on top of a Fermi sea always form a bound state, however small the (attractive) interaction between them [12]. This instability results in the so-called BCS state (coherent state of Cooper pairs), that leads to a gap in the spectrum of excitations, responsible for superconductivity. In the cur-
PACS numbers: 71.35.Gg, 71.36.+c, 71.55.Eq, 74.78.-w

rently available high- $T_{C}$ superconductors (the cuprates), an electron pairing is also thought to be realized through a mediating boson, although probably not the phonon [13]. Excitons have been proposed as suitable mediating bosons to achieve higher critical temperatures of superconductivity in specially designed heterostructures ([14], see [15] for a review). As compared to phonons, the characteristic cutoff energy above which the attractive character of the interaction is lost for the excitons is several orders of magnitude higher and the critical temperature is therefore expected to be sufficiently increased with respect to the

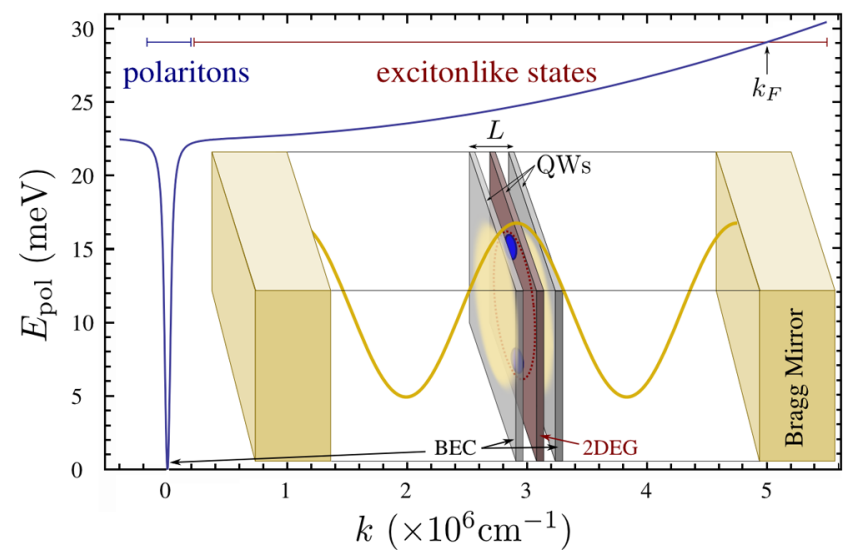

FIG. 1 (color online). The polariton dispersion, on the scale of interest for mediating Cooper pairing at $k_{F}=5 \times 10^{6} \mathrm{~cm}^{-1}$. The photon (electron/hole) effective mass has been taken as $10^{-5} m_{0}\left(0.22 / 1.25 m_{0}\right), m_{0}$ being the electron mass in vacuum, the vacuum Rabi splitting as $\Omega=45 \mathrm{meV}, X^{2}=1 / 2$, and $U A \approx 0.6 \mu \mathrm{eV} \times \mu \mathrm{m}^{2}$. Other parameters are $L=50 \AA, a_{B}=$ $25 \AA, R_{y}=32 \mathrm{meV}, \epsilon \approx 7 \epsilon_{0}$. The peculiar polariton dispersion allows for the coexistence of a BEC at low $k$ that mediates the interaction and of slow excitonlike states at large $k$ providing the retarded electron-electron attraction. In the inset, the scheme of the model microcavity structure with an $n$-doped QW sandwiched between two undoped QWs where the polariton condensate is formed. 
BCS superconductors. One possible implementation of this idea is the so-called sandwich type of superconducting system, consisting of layers of metal and insulator materials where excitons and free electrons are close enough to each other to interact efficiently [16]. Electrons in metal are attracted to each other due to double scatterings involving creation and annihilation of virtual excitons of the semiconductor. The virtual excitons are created due to the nonenergy-conserving scattering process and disappear also due to scattering with free electrons from the metal. However, the probability of such scattering is low, because of the huge energies needed to create virtual excitons. While the metal-insulator sandwiches demonstrated superconductivity at temperatures up to $50 \mathrm{~K}[17,18]$, there is no evidence of the exciton mechanism of superconductivity being realized in this or other systems.

Relying on the recent discovery of high-temperature BEC of exciton polaritons, we propose microcavities as an implementation of the Ginsburg scheme of mediating the interaction between electrons through an excitonlike field [19], using not virtual but real excitons. We show that the strength of electron-electron interactions, mediated by a condensate of exciton polaritons, scales like the density of the polariton condensate $N_{0}$, which can be tuned by the pumping power. This opens the route to optically mediated superconductivity, where the BCS gap value is governed by an optical pumping intensity. We consider a device specially engineered for this purpose (see the inset of Fig. 1), consisting of an $n$-doped QW sandwiched between two undoped QWs at the antinode of the optical field of a microcavity. A polariton condensate is formed in the sandwiching wells, e.g., by optical excitation. The advantage of this design is to maximize the interaction of the twodimensional electron gas (2DEG) with the condensate, which, being delocalized in the structure (thanks to the photonic part of the polariton), can sandwich as a single object the electron gas. In this way, proximity of polaritons and electrons is maximum. Also, a larger number of QWs allows us to reach higher condensate densities of polaritons. In the rest of this Letter, we derive an effective electron-electron Hamiltonian from the electroncondensate interaction, show the existence of an effective attractive potential that grows with the condensate occupancy, and solve numerically the gap equation for the polariton-mediated superconductivity.

The Hamiltonian of the system reads (denoting fermionic operators corresponding to electrons of in-plane momentum $\hbar \mathbf{k}$ by $\sigma_{\mathbf{k}}$, and bosonic operators corresponding to polaritons by $a_{\mathbf{k}}$ ):

$$
\begin{aligned}
H= & \sum_{\mathbf{k}}\left[E_{\mathrm{el}}(\mathbf{k}) \sigma_{\mathbf{k}}^{\dagger} \sigma_{\mathbf{k}}+E_{\mathrm{pol}}(\mathbf{k}) a_{\mathbf{k}}^{\dagger} a_{\mathbf{k}}\right]+\sum_{\mathbf{k}_{1}, \mathbf{k}_{2}, \mathbf{q}}\left[V_{C}(\mathbf{q}) \sigma_{\mathbf{k}_{1}+\mathbf{q}}^{\dagger} \sigma_{\mathbf{k}_{2}-\mathbf{q}}^{\dagger} \sigma_{\mathbf{k}_{1}} \sigma_{\mathbf{k}_{2},}+X V_{X}(\mathbf{q}) \sigma_{\mathbf{k}_{1}}^{\dagger} \sigma_{\mathbf{k}_{1}+\mathbf{q}} a_{\mathbf{k}_{2}+\mathbf{q}}^{\dagger} a_{\mathbf{k}_{2}}\right. \\
& \left.+U a_{\mathbf{k}_{1}}^{\dagger} a_{\mathbf{k}_{2}+\mathbf{q}}^{\dagger} a_{\mathbf{k}_{1}+\mathbf{q}} a_{\mathbf{k}_{2}}\right] .
\end{aligned}
$$

Here $E_{\mathrm{el}}(\mathbf{k})$ and $E_{\mathrm{pol}}(\mathbf{k})$ describe the in-plane dispersion of electrons and exciton polaritons, respectively. The third term describes the direct electron-electron interaction, the fourth the electron-polariton interaction, and the fifth polariton-polariton interactions which are treated within the $s$-wave scattering approximation with strength $U=$ $6 a_{B}^{2} R_{y} X^{2} / A$ (where $a_{B}$ is the exciton Bohr radius, $R_{y}$ the exciton binding energy, $A$ the normalization area, and $X$ is the exciton Hopfield coefficient, whose square quantifies the exciton fraction in the exciton-polariton condensate) [20]. We go beyond BCS theory by including the electronelectron Coulomb interaction $V_{C}$ on top of the electronexciton interactions $V_{X}$. Their respective matrix elements are given by $V_{C}(\mathbf{q})=e^{2} /[2 \epsilon A(|\mathbf{q}|+\kappa)]$ and

$$
\begin{aligned}
V_{X}(\mathbf{q})= & \frac{16 e^{2}}{A \epsilon a_{B}^{3}} \frac{\pi e^{-|\mathbf{q}| L / 2}}{|\mathbf{q}|+\kappa_{\mathbf{q}}}\left\{\frac{1}{\beta_{h}^{2}} \frac{1}{\left(|\mathbf{q}|^{2}+4 / a_{B}^{2} \beta_{h}^{2}\right)^{3 / 2}}\right. \\
& \left.-\frac{1}{\beta_{e}^{2}} \frac{1}{\left(|\mathbf{q}|^{2}+4 / a_{B}^{2} \beta_{e}^{2}\right)^{3 / 2}}\right\},
\end{aligned}
$$

where $\beta_{e(h)}=m_{e(h)} /\left(m_{e}+m_{h}\right)$ [21], $\boldsymbol{\kappa}$ is the screening wavelength in plane for the electrons in the middle QW, which is estimated as $\kappa=m_{e} e^{2} /\left(2 \pi \epsilon \hbar^{2}\right)$ (its weak temperature dependence can be ignored) with $n$ the density of the 2DEG and $\kappa_{\mathbf{q}}=\kappa \exp (-|\mathbf{q}| L / 2)$ is derived from the
Lindhard formula [22] in the geometry of Fig. 1. Here we neglected all exchange terms assuming that the overlap of electron and exciton wave functions is zero. We use the mean-field approximation for the condensate of exciton polaritons, namely, $a_{\mathbf{k}_{1}+\mathbf{q}}^{\dagger} a_{\mathbf{k}_{1}} \approx\left\langle a_{\mathbf{k}_{1}+\mathbf{q}}^{\dagger}\right\rangle a_{\mathbf{k}_{1}}+a_{\mathbf{k}_{1}+\mathbf{q}}^{\dagger}\left\langle a_{\mathbf{k}_{1}}\right\rangle$ and $\left\langle a_{\mathbf{k}}\right\rangle \approx \sqrt{N_{0} A} \delta_{\mathbf{k}, \mathbf{0}}$, with $N_{0}$ the density of polaritons in the condensate. This allows us to obtain the following expression for the Hamiltonian, after diagonalizing the polariton part by means of a Bogoliubov transformation (that leaves the free propagation of electrons and their direct interaction $H_{C}$ invariant):

$$
\begin{aligned}
H= & \sum_{\mathbf{k}} E_{\mathrm{el}}(\mathbf{k}) \sigma_{\mathbf{k}}^{\dagger} \sigma_{\mathbf{k}}+\sum_{\mathbf{k}} E_{\mathrm{bog}}(\mathbf{k}) b_{\mathbf{k}}^{\dagger} b_{\mathbf{k}}+H_{C} \\
& +\sum_{\mathbf{k}, \mathbf{q}} M(\mathbf{q}) \sigma_{\mathbf{k}}^{\dagger} \sigma_{\mathbf{k}+\mathbf{q}}\left(b_{-\mathbf{q}}^{\dagger}+b_{\mathbf{q}}\right)
\end{aligned}
$$

where $E_{\mathrm{bog}}(\mathbf{k})$ describes the dispersion of the elementary excitations (bogolons) of the interacting Bose gas, which is very close to a parabolic exciton dispersion at large $k$,

$$
E_{\mathrm{bog}}(\mathbf{k})=\sqrt{\tilde{E}_{\mathrm{pol}}(\mathbf{k})\left[\tilde{E}_{\mathrm{pol}}(\mathbf{k})+2 U N_{0} A\right]},
$$

where $\tilde{E}_{\mathrm{pol}}(\mathbf{k}) \equiv E_{\mathrm{pol}}(\mathbf{k})-E_{\mathrm{pol}}(\mathbf{0})$ and with the renormalized bogolon-electron interaction strength 


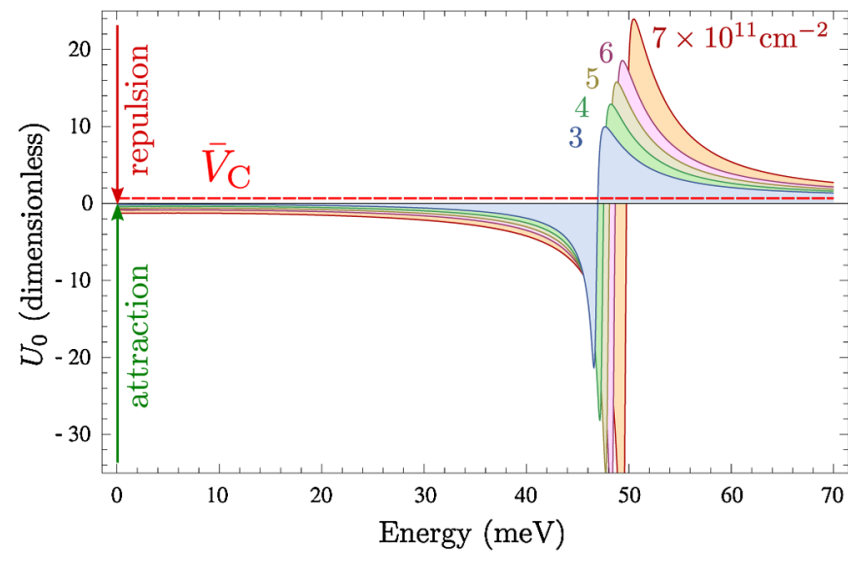

FIG. 2 (color online). The effective electron-electron interaction potential $U_{0}$ [cf. Equation (7)] for different values of the condensate density $N_{0}$ (in $\mathrm{cm}^{-2}$ ). Direct Coulomb repulsion is included (horizontal red dashed line) which fights the fragile retardation effect of excitons. Attraction is restored when the density of the condensate is high enough to overcome Coulomb repulsion, leading to Cooper pairing in the 2DEG.

$$
M(\mathbf{q})=\sqrt{N_{0} A} X V_{X}(\mathbf{q}) \sqrt{\frac{E_{\mathrm{bog}}(\mathbf{q})-\tilde{E}_{\mathrm{pol}}(\mathbf{q})}{2 U N_{0} A-E_{\mathrm{bog}}(\mathbf{q})+\tilde{E}_{\mathrm{pol}}(\mathbf{q})}} .
$$

The last term of Eq. (3) coincides with the Frölich electron-phonon interaction Hamiltonian, which allows us to write an effective Hamiltonian for the bogolonmediated electron-electron interaction. This results in an effective interaction between electrons, of the type $\sum_{\mathbf{k}_{1}, \mathbf{k}_{2}, \mathbf{q}} V_{\text {eff }}(\mathbf{q}, \omega) \sigma_{\mathbf{k}_{1}}^{\dagger} \sigma_{\mathbf{k}_{1}+\mathbf{q}} \sigma_{\mathbf{k}_{2}+\mathbf{q}}^{\dagger} \sigma_{\mathbf{k}_{2}}, \quad$ with $\hbar \omega=$ $E_{\mathrm{pol}}\left(\mathbf{k}_{1}+\mathbf{q}\right)-E_{\mathrm{pol}}\left(\mathbf{k}_{1}\right)$ and the effective interaction strength $V_{\text {eff }}(\mathbf{q}, \omega)=V_{C}(\mathbf{q})+V_{A}(\mathbf{q}, \omega)$, with

$$
V_{A}(\mathbf{q}, \omega)=\frac{2 M(\mathbf{q})^{2} E_{\mathrm{bog}}(\mathbf{q})}{(\hbar \omega)^{2}-E_{\mathrm{bog}}(\mathbf{q})^{2}}
$$

Equation (6) recovers the boson-mediated interaction potential obtained for Bose-Fermi mixture of cold atomic gases [23], in the limit of vanishing exchanged wave vectors. It describes the BEC induced attraction between electrons. Remarkably, it increases linearly with the condensate density $N_{0}$. This represents an important advantage of this mechanism of superconductivity with respect to the earlier proposals of exciton-mediated superconductivity [14-16], as the strength of Cooper coupling can be directly controlled by optical pumping of the exciton-polariton condensate.

The frequency dependence in Eq. (6) describes the retarded character of the mediated electron-electron attraction, which is essential for the stability of a Cooper pair. In general, for the exciton-induced interactions, the retardation is weak as compared to that provided by the phononinduced interactions. In our system the exciton mass is only 6 times heavier than the electron mass, which results in a speed of bogolon excitations 6 times smaller than the Fermi velocity. The weak retardation is compensated by the strong polariton-mediated attraction in the case of a high density of the condensate.

We compute an effective (dimensionless) interaction between electrons by averaging the potential of interaction $V_{\text {eff }}$ over the 2D Fermi sea, that includes the attractive exciton mechanism $\left(V_{A}\right)$ competing with direct Coulomb repulsion $\left(V_{C}\right)[15]$ :

$$
U_{0}(\omega)=\frac{A \mathcal{N}}{2 \pi} \int_{0}^{2 \pi}\left[V_{A}(q, \omega)+V_{C}(q)\right] d \theta
$$

where $\quad q=\sqrt{2 k_{F}^{2}(1+\cos \theta)}, \quad \mathcal{N}=d n /\left.d E\right|_{E_{F}}=$ $m_{e} /\left(\pi \hbar^{2}\right)$ is the density of states at the Fermi surface of the 2DEG. The shapes of $U_{0}(\omega)$ for various polariton densities are shown in Fig. 2. Coulomb repulsion contributes an overall (frequency independent) repulsion $\bar{V}_{C}$, of order 0.67 (it is dimensionless) for our parameters. This repulsion is detrimental to the exciton mechanism, which has a fragile retardation effect compared to metals. At the density of $N_{0}=2 \times 10^{11} \mathrm{~cm}^{-2}$, for instance, the effective electron-electron potential is dominated by Coulomb repulsion and thus becomes repulsive again at long times. It exhibits attraction only in a narrow region of frequencies, that is not sufficient to bind Cooper pairs. At higher densities, retardation extends over longer time, like in the BCS picture. We compute the average of $V_{A}$ numerically and solve the gap equation for $U_{0}$

$$
\Delta(\xi, T)=-\int_{-\infty}^{+\infty} \frac{U_{0}\left(\xi-\xi^{\prime}\right) \Delta\left(\xi^{\prime}, T\right) \tanh \left(E / 2 k_{B} T\right)}{2 E} d \xi^{\prime}
$$

by iteration, where $E=\sqrt{\Delta\left(\xi^{\prime}, T\right)^{2}+\xi^{\prime 2}}$. In the inset of Fig. $3, \Delta(0, T)$ is displayed for $N_{0}=6 \times 10^{11} \mathrm{~cm}^{-2}$. A gap is open on the Fermi sea, that results in the Cooper instability that drives the ground state to a BCS-like state. The gap is suppressed by temperature, but its value and the critical temperature $T_{C}$, at which the gap vanishes, strongly depend on $N_{0}$, as shown in Fig. 3. We have used the numerical parameters typical of GaN-based microcavities (see caption of Fig. 1), where polariton lasing at room temperature has been recently reported [24]. The free electron concentration has been chosen as $n \approx$ $4 \times 10^{12} \mathrm{~cm}^{-2}$, so that $k_{F}=5 \times 10^{6} \mathrm{~cm}^{-1}$ falls in the exciton part of the polariton dispersion (cf. Figure 1). The lateral size of the polariton-mediated Cooper pairs can be estimated as $\chi_{C} \approx\left(2 \pi \hbar^{2} k_{F}\right) /\left(m_{e} \Delta(0,0)\right)$. At the highest power reported here, $\chi_{C} \approx 196 \AA$ is well above the average distance between electrons, $n^{-1 / 2} \approx 25 \AA$. The parameters typical of the GaAs-based microcavities give essentially similar results, while in GaAs-based cavities the polariton BEC cannot exist at temperatures higher than several tens of kelvin, so that superconducting $T_{C}$ would not exceed this value.

The critical temperature for superconductivity is zero for low exciton-polariton concentrations because Coulomb repulsion of electrons prevents formation of the Cooper 


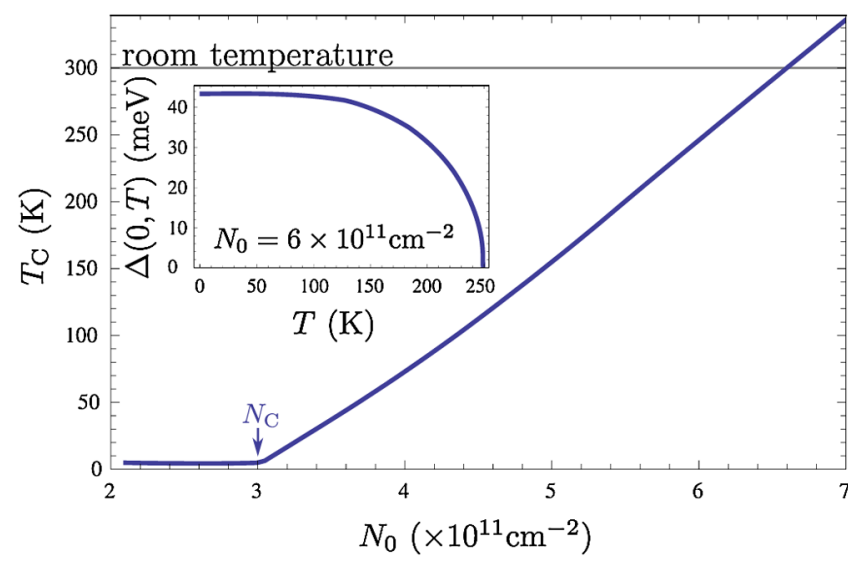

FIG. 3 (color online). Critical temperature of superconductivity as a function of the exciton-polariton condensate density $N_{0}$. Inset: Closing of the gap at the energy of the Fermi sea for $N_{0}=$ $6 \times 10^{11} \mathrm{~cm}^{-2}$.

pairs. The electron-electron interaction is repulsive at low frequencies in this case. Above a critical density, which is slightly below $N_{C} \approx 3 \times 10^{11} \mathrm{~cm}^{-2}$ in our model system, the superconductivity becomes possible. The electronelectron interaction strength dependence on the polariton density leads to a roughly linear increase of the critical temperature $T_{C}$ with $N_{0}$ in this region. This is characteristic for systems with strong electron-electron interactions. Tuning the condensate density with external power allows a transition to the superconducting regime, up to very high temperatures (limited by the Mott transition for the exciton-polariton condensate expected at about $N_{0}=$ $10^{12} \mathrm{~cm}^{-2}$ in GaN-based microcavities [8]); with a GaN structure, this would enable superconductivity up to room temperature.

In order to evidence experimentally the light-mediated superconductivity, one could measure the in-plane differential photoconductivity of the microcavity. The carriers need to be injected in the $n$-doped quantum well from metallic contacts. The polariton condensate may be created by resonant optical pumping. The sign of differential photoconductivity is expected to change from negative to positive at the onset of superconductivity.

In conclusion, we propose a new mechanism to achieve superconductivity, based on microcavity polaritons. A Bose-Einstein condensate of polaritons is offered as a mediator for the interactions between electrons in a specially engineered device. The magnitude of attraction increases linearly with the condensate density, allowing for an external control of the binding energy, and therefore of the critical temperature. With devices that have demonstrated polariton BEC up to room temperature, our findings suggest that exciton polaritons could be promising candidates to achieve high-temperature superconductivity in a semiconductor structure, with critical temperatures only limited by that of the BEC.

We thank T. Taylor for help in numerical modeling. F.P.L. and A. V.K. acknowledge support from the EPSRC and A. V. K. from EU e-I3 ETSF project 211956.

[1] A. Kavokin, J. J. Baumberg, G. Malpuech, and F. P. Laussy, Microcavities (Oxford University Press, Oxford, 2007).

[2] P. G. Savvidis, J. J. Baumberg, R. M. Stevenson, M. S. Skolnick, D. M. Whittaker, and J. S. Roberts, Phys. Rev. Lett. 84, 1547 (2000).

[3] H. Deng, G. Weihs, C. Santori, J. Bloch, and Y. Yamamoto, Science 298, 199 (2002).

[4] J. Kasprzak et al., Nature (London) 443, 409 (2006).

[5] A. Amo, J. Lefrère, S. Pigeon, C. Adrados, C. Ciuti, I. Carusotto, R. Houdré, E. Giacobino, and A. Bramati, Nature Phys. 5, 805 (2009).

[6] A. Amo et al., Nature (London) 457, 291 (2009).

[7] J. J. Baumberg et al., Phys. Rev. Lett. 101, 136409 (2008).

[8] A. Kavokin, G. Malpuech, and F. P. Laussy, Phys. Lett. A 306, 187 (2003).

[9] P. G. Lagoudakis, M. D. Martin, J. J. Baumberg, A. Qarry, E. Cohen, and L. N. Pfeiffer, Phys. Rev. Lett. 90, 206401 (2003).

[10] J. Bardeen, L. N. Cooper, and J. R. Schrieffer, Phys. Rev. 106, 162 (1957).

[11] J. Bardeen, Rev. Mod. Phys. 23, 261 (1951).

[12] L. N. Cooper, Phys. Rev. 104, 1189 (1956).

[13] F. Giustino, M. L. Cohen, and S. G. Louie, Nature (London) 452, 975 (2008).

[14] W. A. Little, Phys. Rev. 134, A1416 (1964).

[15] V.L. Ginzburg, On Superconductivity and Superfluidity (Springer, New York, 2009).

[16] D. Allender, J. Bray, and J. Bardeen, Phys. Rev. B 7, 1020 (1973).

[17] C. Aruta, G. Ghiringhelli, C. Dallera, F. Fracassi, P. G. Medaglia, A. Tebano, N. B. Brookes, L. Braicovich, and G. Balestrino, Phys. Rev. B 78, 205120 (2008).

[18] A. Gozar, G. Logvenov, L. F. Kourkoutis, A. T. Bollinger, L. A. Giannuzzi, D. A. Muller, and I. Bozovic, Nature (London) 455, 782 (2008).

[19] High Temperature Superconductivity, edited by V. Ginzburg and K. Kirzhnits (Pergamon, New York, 1977).

[20] F. Tassone and Y. Yamamoto, Phys. Rev. B 59, 10830 (1999).

[21] C. Ciuti, V. Savona, C. Piermarocchi, A. Quattropani, and P. Schwendimann, Phys. Rev. B 58, 7926 (1998).

[22] H. Haug and S. W. Koch, Quantum Theory of the Optical and Electronic Properties of Semiconductors (World Scientific, Singapore, 1990).

[23] M. J. Bijlsma, B. A. Heringa, and H. T. C. Stoof, Phys. Rev. A 61, 053601 (2000).

[24] G. Christmann, R. Butté, E. Feltin, J.-F. Carlin, and N. Grandjean, Appl. Phys. Lett. 93, 051102 (2008). 